\title{
12 Molecular Visualization of an Enzyme, Acetylcholinesterase
}

\subsection{Learning Objectives}

The goal of this laboratory is to analyze some of the major structural elements of an important enzyme, acetylcholinesterase (AChE). To do this, you will use a common structural visualization program and correlate AChE structural elements with the enzyme mechanism. You will be using Chimera, a state-of-the-art molecular visualization program provided by the National Science Foundation through the University of California, San Francisco. This free program is available at http://www.cgl.ucsf.edu/chimera/.

To provide a quick overview of the program, we will look at a multi-drug resistance efflux pump - the protein that is controlled by the riboswitch you studied. You will then use this program to analyze the enzyme, acetylcholinesterase.

\subsection{Introduction and Background}

Acetylcholinesterase (AChE) destroys the nerve transmitter, acetylcholine by hydrolysis.<smiles>CC(=O)O[PH2+][CH2+]C(=O)OCC[N+](C)(C)[C@@H](O)C[18OH]</smiles>

AChE is one of the most efficient enzymes in nature - in some ways, a "perfect" enzyme. Amino acid side chains at the active site are precisely arrayed to force bonding changes in the acetylcholine (Fig. 12.1).

Overall, the electrons are pushed toward the ester carbonyl forming a covalent intermediate between the reactant and the enzyme (Fig. 12.2).

Acetylcholinesterase's finely tuned mechanism provides a good illustration of some common catalytic processes:

- Covalent Intermediate Catalysis - The activation energy is lowered because an especially reactive active site group attacks and forms a new covalent bond to the reactant;

- Acid-Base Catalysis - The activation energy is lowered because active site groups force electron (and proton) movement.

- This week you will examine this enzyme at a molecular level. Next week you will study the enzyme kinetics of acetylcholinesterase. 


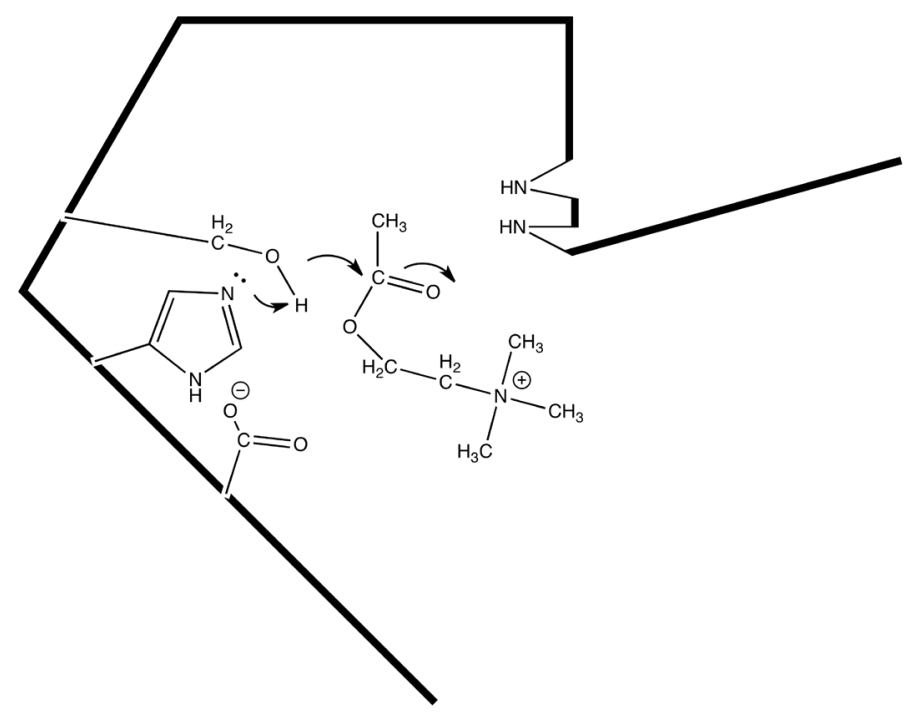

Figure 12.1: A schematic of the acetylcholinesterase active site showing the electron movement during the initial nucleophilic attack of acetylcholine: The alcohol acts as a nucleophile attacking the ester carbonyl carbon. This alcohol is a strong nucleophile because the adjacent imidazole ring changes the alcoholic hydroxyl group to an alkoxide. In other words, the imidazole acts as an especially strong base. It can act in this way, because of the adjacent carboxylate negative charge.

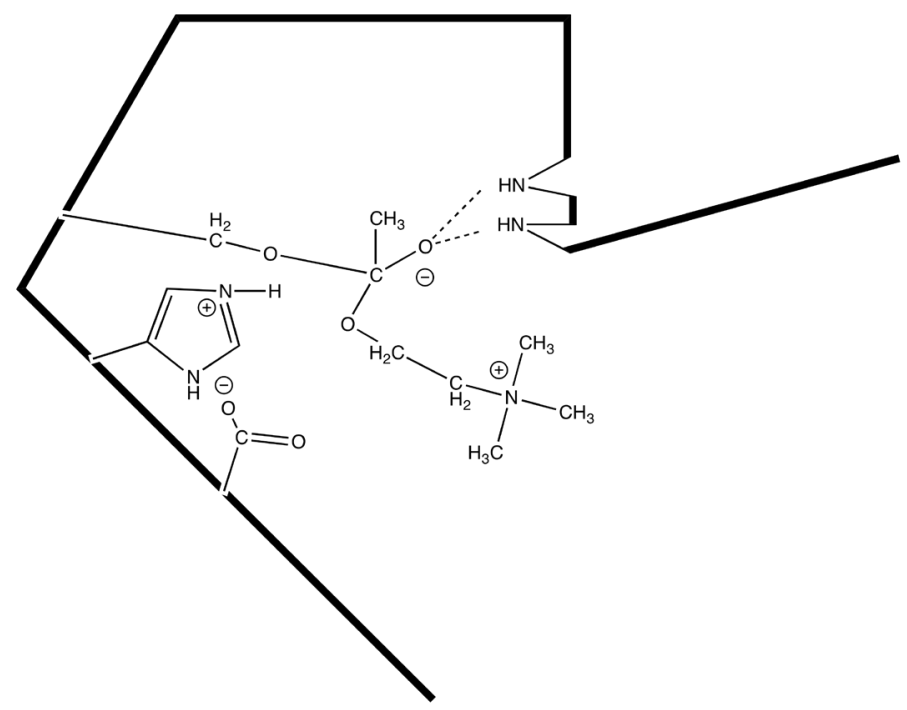

Figure 12.2: A schematic of the acetylcholinesterase active site showing the covalent intermediate formed following the initial nucleophilic attack: Note that the negative charge that forms on the carbonyl oxygen is stabilized by hydrogen bonding with "-NH" groups. Once this covalent intermediate forms, choline rapidly leaves the active site and water enters to complete the reaction. 


\subsection{Introduction to Molecular Visualization Using the Program Chimera}

The visualization program Chimera will be introduced to you by using the structure of the multi-drug resistance (MDR) efflux pump protein. This is the protein that is up-regulated by a riboswitch in response to antibiotics like tetracycline. The protein then pumps the antibiotics out of the bacteria allowing the cells continued growth. Because the MDR efflux pump eliminates many different drugs, the bacteria become multi-drug resistant.

The MDR efflux pump is an integral membrane protein. As such, the protein structure has some common membrane protein structural characteristics. First, transmembrane helices comprise the bulk of the protein. These helices stretch the width of the lipid bilayer and expose hydrophobic amino acids to the lipid bilayer interior. Hydrophilic amino acids are located where the protein meets the lipid bilayer surface, both on the outside of the cell as well as on the inside.

The instructions for using Chimera follow this format:

Italics indicates that you should go to a Menu or a Command line or the Cursor

Bold... tells you which menu to access

...Bold indicates a selection from the accessed menu

Bold indicates a command

1. First, download the protein data. Structural data for macromolecules is available from a central data bank, the RCSB Protein Data Bank. Every structure is given its own unique code. For example, the multi-drug resistance efflux pump data set used here is "2GFP."

\section{Menu: File... Fetch by ID \\ type in box $2 \mathrm{GFP}$ \\ click Fetch button}

2. The command line can be used for very specific changes. To bring up the command line (or hide it) go to the "Tools" menu, "General Controls" sub-menu.

Command: del :.b

Command: focus

3. The cursor (mouse) provides for quick changes in the protein view.

Cursor: left mouse button + moving cursor = rotates protein

Cursor: ctrl + left mouse button + moving cursor $=$ selects protein

Cursor: right mouse button + moving cursor $=$ changes size of protein

Cursor: ctrl + right mouse button + moving cursor $=$ translates protein

Place cursor over the protein to identify specific amino acids 
4. The "Presets" menu gives choices for common ways to represent the protein.

Мепи: Presets... Interactive 1

Мепu: Presets... Interactive 2

Мепu: Presets... Interactive 3

Menu: Presets... Interactive 4

5. The "Select" menu specifies what part of the protein will be changed by the "Actions" menu. Try the following three examples:

a. Example \#1: A chain of amino acids linked by peptide bonds (a polypeptide) is selected and any action now will apply to that chain (e.g., changing color).

Menu: Select... Chain... A

Menu: Actions... Color... ?

b. Example \#2: All amino acids that carry a negatively charged side chain at neutral $\mathrm{pH}$ (i.e., carboxylates) are selected and all the atoms/bonds in the side chains are (a) shown and (b) colored by element.

мепи: Select... Clear Selection

Мепu: Select... Residue... amino acid categories... negative

Menu: Actions... Atoms/bonds... show

Мenu: Actions... Color... by element

c. Example \#3: All amino acids that carry a positively charged side chain at neutral $\mathrm{pH}$ are selected and all the atoms/bonds in the side chains are shown and colored by element. The atoms are then shown as actual size (sphere). Finally, the protein is shown as a solid object with a surface, as it would actually appear.

Мепu: Select... Selection Mode (replace)... append

Мепu: Select... Residue... amino acid categories... positive

Menu: Actions... Atoms/bonds... show

Мenu: Actions... Color... by element

Меnu: Actions... Atoms/Bonds... sphere

Мепu: Actions... Atoms/Bonds... hide

Мепu: Select... Clear Selection

Menu: Select... Chain... A

Мепu: Actions... Surface... show

6. The tools menu can provide further information about protein properties.

Мепu: Tools... Surface/Binding Analysis... Coulombic Surface

Coloring

click OK button

мепu: Actions...Surface...Hide 


\title{
Menu: Tools... Depiction... Color Secondary Structure click OK button \\ Мепu: File... Close Session
}

\subsection{Analysis of Acethylcholinesterase Using the Computer Visualization Program Chimera}

The following series of tasks help you learn how to use computer visualization software to better understand how the enzyme acetylcholinesterase works. Follow the instructions below to answer the questions.

1. The overall structure of acetylcholinesterase. Proteins are stabilized by secondary structures, commonly either $\beta$-pleated sheets or $\alpha$-helices.

\author{
Мепu: File... Fetch by ID \\ type in box $1 \mathrm{AMN}$ \\ click Fetch button \\ Menu: Tools... Depiction... Color Secondary Structure \\ click OK button \\ Command: del : $\mathrm{HOH}: \mathrm{SO} 4$
}

a. (2 pts.) How many strands are included in each of the two $\beta$-pleated sheets?

b. (3 pts.) Identify the two longest $\alpha$-helices by listing the abbreviations for the amino acids at the beginning and the end of each helix. (Placing your cursor over a spot on the protein will cause the abbreviation to be shown.) How many amino acid residues are in each helix? [Hint: the amino acids are numbered consecutively.]

2. Substrate Analog (NAF) at the Active Site. Active sites are often marked or labeled with a substrate analog. This is a substrate-like molecule that reacts incompletely at the enzyme active site. It remains bound to the enzyme and marks some of the catalytic amino acid side chains. In this case the substrate analog forms a structure like the tetrahedral intermediate shown in the Introduction and Background. 


\author{
Мепи: Select... Residue... NAF \\ Menu: Actions... Atoms/Bonds... Show \\ Мепu: Select... Invert (all models) \\ Мепu: Actions... Ribbon... Hide \\ Мепu: Actions... Atoms/Bonds... Hide \\ Command: display: 200 \\ Command: focus
}

a. (4 pts.) Draw the structural formula for NAF connected to an atom of an amino acid side chain (treat the rest of the side chain as an " $R$ " group). This structure contains two ionic charges (one positive and one negative). Can you locate these charges? Mark each with either a “+” or a “-.”

b. (4 pts.) NAF forms a covalent intermediate with the enzyme just as the natural substrate, acetylcholine, does. This intermediate is called a "tetrahedral intermediate.” Why do you think it is given that name? Be as specific as possible.

3. Noncovalent Interactions between the Active Site and the Substrate Analog. Van der Waals contacts between the protein and the substrate analog are very common.

\author{
Мепи: Select... Clear Selection \\ Мепu: Select... Residue... NAF \\ Мепu: Tools... Structure Analysis... Clashes/Contacts \\ click Designate button \\ click Contact button \\ check Select \\ uncheck Draw pseudobonds of color \\ check Color \\ click $\mathbf{O K}$ button
}


a. (3 pts.) How many atoms of NAF are directly in contact with the protein? Out of a total of how many atoms in NAF? [Atoms marked in red are in direct contact with the protein.] Mark each atom that is in contact with an asterisk (*) in your NAF structure in question \#3.

4. Visualize the amino acids that immediately surround the substrate analog click on Graphics Window then hit the arrow up key [This will select the complete NAF.]

Мепu: Actions... Atoms/Bonds... Show

Меnu: Actions... Atoms/Bonds... Sphere

Command: focus

Мепu: Select... Clear Selection

Мепu: Select... Residue... NAF

Menu: Actions... Color... choose a color that is easy to see

a. (4 pts.) Does the NAF have any "wiggle room" when bound at the active site? How do you think that this might aid enzyme catalysis?

5. Select active site amino acids that help catalyze the reaction.

Мепu: Actions... Atoms/Bonds... Stick

Menu: Actions... Atoms/Bonds... Color... by element

Мепu: Select...Invert (all models)

Мепи: Actions... Atoms/Bonds... Stick

Menu: Actions... Atoms/Bonds... Color... by element

Command: show :118: 119: 200: 327: 4 : NAF

Command: focus 
a. (3 pts.) Identify the $\alpha$-carbon atom for each amino acid. The $\alpha$-carbon lies between a carboxyl " $\mathrm{C}=\mathrm{O}$ " and an " $\mathrm{N}-\mathrm{H}$ " along the protein backbone. The side chain for each amino acid is also connected to the $\alpha$-carbon. (The side chain for a glycine amino acid is simply a " $\mathrm{H}$ " and so it is not shown in the structure.) One side chain is covalently bonded to NAF. Move your cursor to the $\alpha$-carbon to learn the abbreviated name for this amino acid. Use the list of amino acid structures below to identify this amino acid and draw its side chain structure.

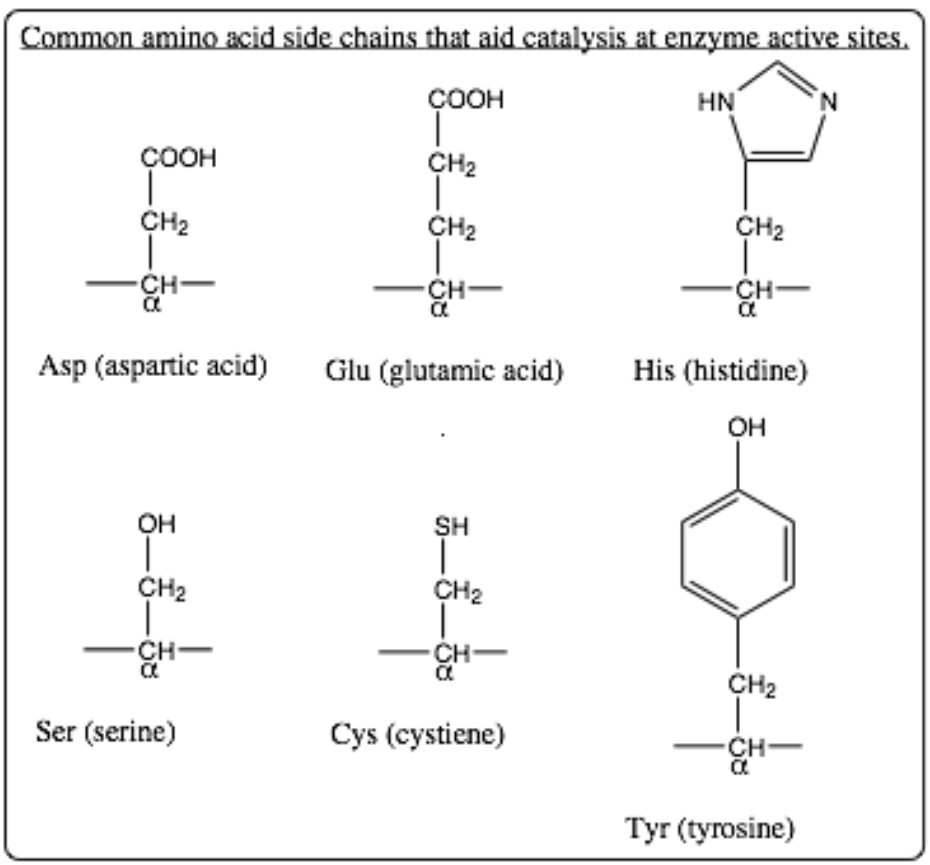

b. (4 pts.) Draw structures for all other amino acid side chains shown at this active site. Identify each amino acid as well. At the catalytic step shown by this structure determined by x-ray crystallography, two of the amino acid side chains carry an ionic charge (one positive and one negative). Identify the charged side chains and give them their appropriate charges.

\section{Menu: Tools...Structural Analysis... FindHbond click OK button}


c. (3 pts.) The blue lines show H-bonds and the H-bonded atoms are close enough together to catalyze reactions.

Two hydrogen bonds that connect to NAF are not from amino acid side chains. Describe or identify the groups from which these $\mathrm{H}$-bonds originate.

Measure the distance between atoms along the blue lines (H-bonds). [Place the cursor over a H-bond. A box will appear detailing the atom within each residue that is involved in H-bonding as well as the distance of the H-bond.] Identify the atoms and give distances.

Ctrl + Left click and drag over all atoms to select

Menu: Actions... Atoms/Bonds... sphere

d. (4 pts.) Note that where H-bonding occurs, the electron clouds are overlapping. What does this mean in terms of bonding?

6. Location of the Active Site with respect to the Overall Protein Structure - the active site can often be recognized as a "pit" or "crevasse" or an "indentation" in the enzyme surface.

Мепu: Select... Chain... A

Мепu: Actions... Ribbons... Show

Мепu: Select... Clear Selection

Command: focus

Menu: Actions... Surface... Show

Мепи: Select... Residue... NAF

Мепu: Actions... Colors... green 
a. (4 pts.) Locate NAF. This is a marker for the location of the active site. Describe the active site location relative to the bulk protein. Might this location cause problems for the enzyme? Briefly explain.

7. Protein Surface Charges and Catalysis

Command: del:NAF [This will leave a "hole" in the active site.]

Menu: Tools... Surface/Binding Analysis... Coulombic Surface Coloring choose 11 for number of colors/values click $\mathbf{O K}$ button [Close any error messages. This calculation may take a minute.]

a. (3 pts.) Describe the location of the predominately blue region (positively charged region) with respect to the active site. How might this relative location help catalysis?

b. (3 pts.) Describe the location of the predominately red region (negatively charged region) with respect to the active site. How might this relative location help catalysis?

8. Protein Motion During Catalysis - proteins are commonly pictured as static, but they flex and bend as part of their function.

Мепu: File... Close Session

Menu: File... Fetch by ID

type in box 2v96

click Fetch button

Мenu: Presets... Interactive 1 (ribbons)

Command: del :.b 


\author{
Menu: File... Fetch by ID \\ type in box 2va9 \\ click Fetch button \\ Command: del :.b
}

Menu: Tools... Structure Comparison... Match Maker

highlight reference structure 2V96

check After superposition, compute structure-based multiple sequence alignment

click Apply button

check Iterate superposition/alignment... in window that opens

check Iterate alignment until convergence

click $\mathbf{O K}$ button

Меnu: Tools... Structure Comparisons... Morph Conformations

click Add button

double click on 2V96.pdb (\#0) in new window

double click on 2VA9.pdb (\#1) in new window

double click on 2V96.pdb (\#0) in new window

select Action on Create: hide Conformations

click Create button

Command: focus

MD Movie: Molecular Movement... [This is a new window that opens.]

click Play button

a. (3 pts.) Can you find motion in this protein as it catalyzes the reaction? What type of structures move - a loop, an $\alpha$-helix, a $\beta$-pleated sheet?

9. Protein Motion at the Active Site

Command: display \#2:200

(Note that this marks the active site using one of the active site amino acids.)

Command: display \#2: 70: 74: 84: 121: 279 290 330: 330:

(Note that these amino acids line the "tunnel" into the active site.)

a. (3 pts.) These motions have been described as "breathing" of the "tunnel." Why would you expect motions like this in the tunnel that leads to the active site? Briefly explain. 


\section{Notes to Instructor}

This laboratory allows the students to "try out" the Chimera program - to experiment with different settings and commands. We find that Chimera is relatively user-friendly and forgiving. Students are encouraged to download this free software for their own use. Chimera may be used with at-home assignments based on the lecture portion of a biochemistry course as well. This is a good opportunity to connect with topics covered in lecture as well as the laboratory. 


\section{Molecular Visualization of Acethylcholinesterase Prelab}

Using any common sources (internet, biochemistry textbook, etc.), answer the following questions concerning the general characteristics of the enzyme, acetylcholinesterase.

1. (3 pts.) What reaction is catalyzed by acetylcholinesterase? Show the structures of reactants and products.

2. (3 pts.) In general terms how is acetylcholinesterase important to neuronal transmission across a synapse?

3. (3 pts.) Where is acetylcholinesterase located within the synaptic space? For example, is acetylcholinesterase found on the presynaptic membrane or on the postsynaptic membrane or as a soluble enzyme in the synaptic cleft or ... ? In general, is this enzyme free to diffuse away from the synapse? Briefly explain. 


\section{Acetylcholinesterase Characteristics Worksheet}

Using any common sources (internet, biochemistry textbook, etc.), answer the following questions concerning the general characteristics of the enzyme, acetylcholinesterase.

1. (2 pts.) Is the natural form of acetylcholinesterase a single polypeptide chain (a monomer)? Or is acetylcholinesterase found as a polymer? Explain.

2. (2 pts.) Briefly describe the steps acetylcholine takes, starting from being in the presynaptic vesicle and ending when acetylcholine is reacted by acetylcholinesterase.

3. (3 pts.) Many nerve gasses (e.g., sarin (GB), soman (GD)) were designed to impact acetylcholinesterase. Specifically, what do these nerve gasses do to this enzyme? How does the change in acetylcholinesterase (brought about by nerve gasses) affect nerve transmission?

4. (3 pts.) Many common insecticides (e.g., malathion, Sevin) also affect acetylcholinesterase. Also some drugs used to treat diseases such as Alzheimer's and myasthenia gravis target acetylcholinesterase. Examples of these drugs include physostigmine (eserine), neostigmine, and pyridostigmine. How is the activity of acetylcholinesterase impacted by these insecticides or drugs? How does this change in acetylcholinesterase affect nerve transmission? 